\title{
Electromagnetic Linear Actuator providing High Force Density per Unit Area without Position Sensor as a Tactile Cell
}

\author{
Takuya Noguchi* Student Member, Sakahisa Nagai* Student Member \\ Atsuo Kawamura,** Fellow
}

(Manuscript received Sep. 13, 2017, revised Dec. 18, 2017)

\begin{abstract}
A novel, very small linear actuator that can realize high force density per cross section without position and force sensors is proposed. This actuator is used in a two-dimensional tactile display, which consists of many small onedimensional actuators with position and force controllability. The proposed actuator has 3 layer stators, and can achieve a high electro-magnetic force. As a result, a force density of $4 \mathrm{mN} / \mathrm{mm}^{2}$ is obtained with a cross section of $4 \mathrm{~mm} \times 4 \mathrm{~mm}$ and a moving stroke of $1 \mathrm{~mm}$.
\end{abstract}

Keywords: actuator, tactile display, haptics

\section{Introduction}

A tactile sensor is considered to be used as a next generation human interface for mass media after visual and audio sensations are matured. The literatures of this research field have been rapidly increased depending on development of position and force research ${ }^{(1)-(4)}$. These are mainly concerning one-dimensional tactile actuation. Recently, device oriented researches for transmission of two-dimensional tactile sensation have been also conducted in various fields ${ }^{(5)-(11)}$. For transmission of tactile sensation, it is necessary to simultaneously transmit position and force information ${ }^{(1)}$. Depending on difference in speed of information transmission and the volume of tactile actuator there are approximately two types of applications. One is, for example, a Braille device for blind people ${ }^{(12)(13)}$ which controls only the positions of dotted points with an open loop, and it can recognize characters by 9 dotted position information. The speed and volume of information in this field is low. Another application is concerning a two-dimensional tactile display, which needs fast and accurate control of the position and force ${ }^{(5)(6)(14)}$. Hereafter, this paper focuses on the actuator aiming at realization of a two-dimensional tactile display. The smallest device that can achieve fast and accurate position and force control in high responsiveness is required for a cell element of tactile sensor and this is defined " tact-cell" in this paper, which should be a small and compact enough for this purpose. Figure 1 shows one example of the illustration of tact-cell's. The piezoelectric bimorph actuator utilizing the piezoelectric effect was proposed ${ }^{(7)}$ in which the shape is deformed by applying a voltage. The structure is a board shape for increase of the deformation. Thus, it is difficult to arrange it into the plane shape as shown in Fig. 1. The SMA

\footnotetext{
* Division of Electrical and Computer Engineering, Yokohama National University

79-5, Tokiwadai, Hodogaya-ku, Yokohama 240-8501, Japan

** Kanagawa Institute of Industrial Science and Technology

3-2-1, Sakado, Takatsu, Kawasaki 213-0012, Japan
}

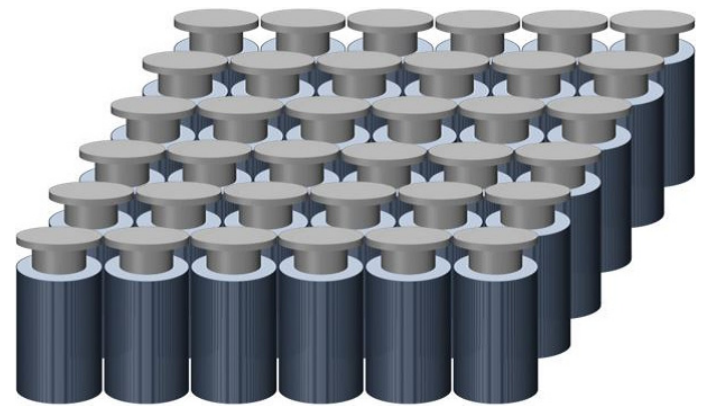

Fig. 1. Illustration of $6 \times 6$ tact-cell's ${ }^{(6)}$

film actuator was proposed in (8) that uses the shape memory effect in which the original geometrical shape comes back when the temperature of the devise reaches a certain thresh hold level. The response time is several seconds because the shape change is caused by heat. The pneumatic actuator was proposed in (9) utilizing pneumatic pressure. This actuator consists of an elastic layer, flow channel and air compressor. The elastic layer is deformed by air pressure. The device becomes too large for tact-cell with an air pump obtaining the air pressure. The magnetic micro actuator ${ }^{(10)}$ was proposed utilizing the repulsive force of the magnet and the electromagnetics. The output is $7.5 \mathrm{mN}$ at the maximum, which is small compared with the $50 \mathrm{mN}$ which is the desired output ${ }^{(9)}$. Furthermore, the literatures ${ }^{(7)-(10)}$ do not mention the force and position control method. The simultaneous sensorless control of position and force is realized by using a solenoid actuator ${ }^{(14)}$. However, the size of this actuator is $15 \mathrm{~mm} \times 10 \mathrm{~mm} \times 43.5 \mathrm{~mm}$, the maximum generated force is about $35 \mathrm{mN}$, and the operation stroke is about $1 \mathrm{~mm}$. It is desirable to be smaller size and bigger generated force for tact-cell. Therefore, the authors proposed a compact and lightweight linear actuator ${ }^{(11)}$ and showed the possibility of simultaneous control of position and force. Figure 2 shows comparison of mover stroke and maximum force in Refs. (5) (7)-(11). And the characteristics of various actuator in Fig. 2 and input conditions are summarized in Table 1. As 


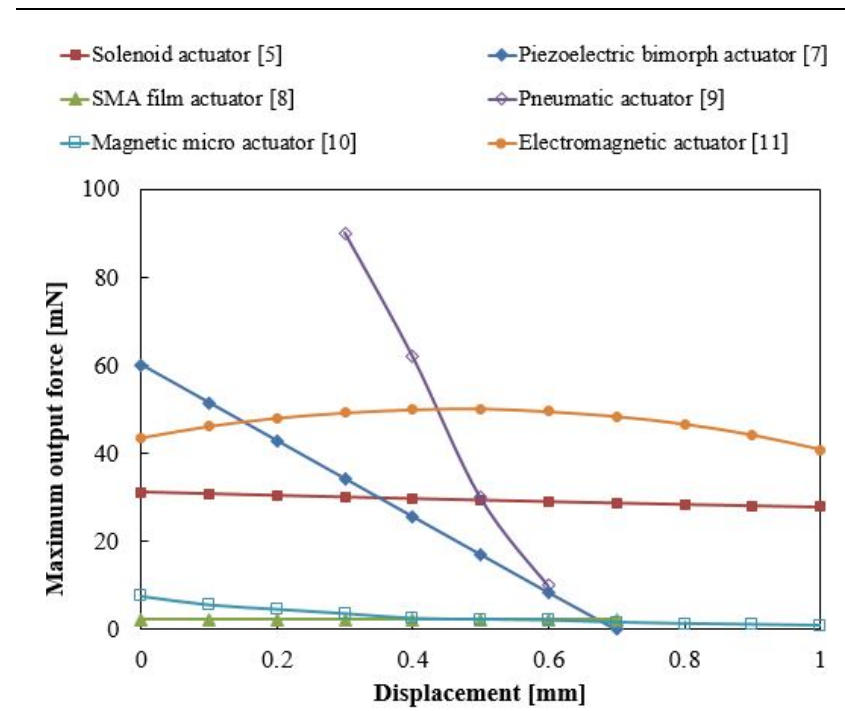

Fig. 2. Relation between maximum output force and displacement of various actuators

Table 1. The characteristics of various actuator and input conditions in Fig. 2

\begin{tabular}{c|c|c|c}
\hline Actuator & surface area $\left(\mathrm{mm}^{2}\right)$ & responce speed & input conditions in Fig. 2 \\
\hline Solenoid $^{(5)}$ & 150.0 & fast & $0.1 \mathrm{~A}$ \\
Piezo $^{(7)}$ & 21.3 & very fast & $150 \mathrm{~V}$ \\
SMA film $^{(8)}$ & 10.5 & slow & $0.6 \mathrm{~A}$ \\
Pneumatic $^{(9)}$ & 569.3 & slow & $100 \mathrm{kPa}$ \\
Magnetic micro $^{(10)}$ & 4.0 & fast & $0.8 \mathrm{~A}$ \\
Electromagnetic $^{(11)}$ & 16.0 & fast & $0.2 \mathrm{~A}$ \\
\hline
\end{tabular}

$$
\begin{aligned}
& \rightarrow \text {-Solenoid actuator [5] } \\
& \text {-SMA film actuator [8] } \\
& \leadsto \text { Piezoelectric bimorph actuator [7] } \\
& \text { —Magnetic micro actuator [10] } \\
& \rightarrow \text { Pneumatic actuator [9] }
\end{aligned}
$$

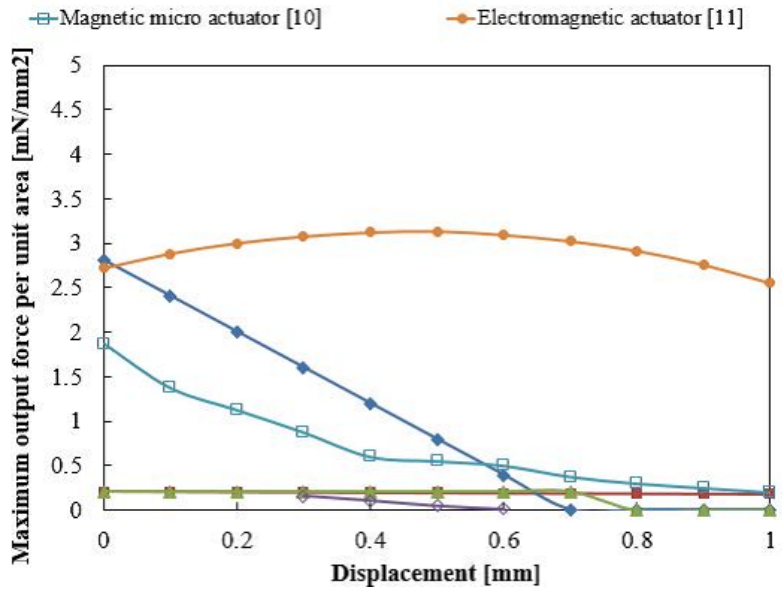

Fig. 3. Relation between maximum output force per unit area and displacement of various actuators

described above, the surface area of the actuator is also important as the tact-cell, thus the relation between maximum output force per unit surface area and stroke of various actuators are shown in Fig. 3. As a result, the actuator ${ }^{(11)}$ is superior to others for a tact-cell. If the output force can be further increased, the performance of this kind of linear actuators is improved as a tact-cell. Therefore, the primary objective of this paper is to increase the output force while maintaining the size. In Ref.(11), the stator was divided into two layers and the thrust force was improved. In this paper, the stator is divided into three layers and laterally stacked in $\mathrm{N}$ and $\mathrm{S}$ alternately location with a multiple pole. The detailed structure

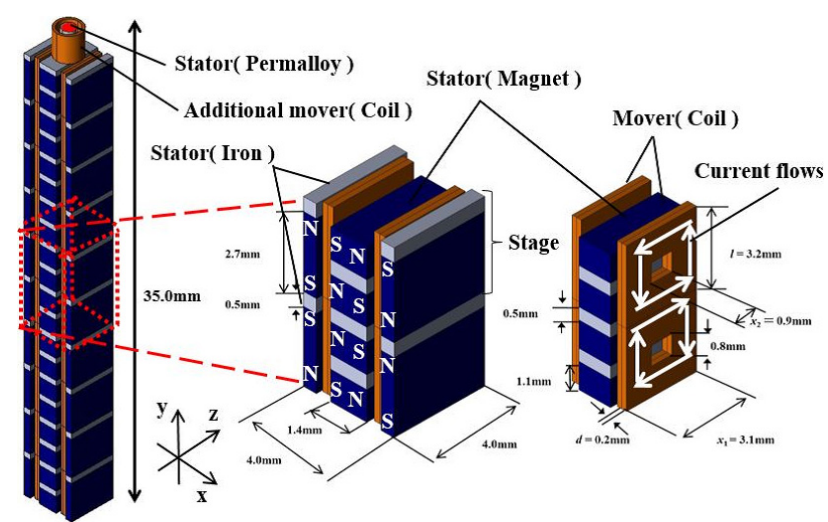

Fig. 4. Proposed actuator

Table 2. Material used in proposed actuator

\begin{tabular}{c|c}
\hline Part & Material \\
\hline Magnet & Neodymium magnet N-50 \\
Mover & Copper \\
Iron & Carbon steel S50C \\
Permalloy & permalloy (PC) \\
\hline
\end{tabular}

is explained in Section 2-1. In general, efficiency of electromagnetic force devise is equal to or higher than the actuators in the past literatures ${ }^{(5)(7)-(11)}$.

The paper is organized as follows. Firstly a novel electromagnetic linear mini-actuator is proposed. The principle of force generation and position estimation are presented in Section 2. The thermal analysis is conducted in Section 3. The experimental results are reported in Section 4. At the end, a conclusion is given and future perspectives are discussed in section 5 .

\section{Proposed Actuator}

2.1 Structure of Proposed Actuator This section explains the structure of proposed actuator. Figure 4 shows a general view and the structure of the proposed actuator. The brown part shows the mover. The blue and silver part shows the stator structure. The actuator consists of iron, magnet, permalloy and coil. The materials used in the proposed actuator are listed in Table 2.

The mover is driven in the vertical direction and the stroke is $1.0 \mathrm{~mm}$. The size per stage is $4.0 \mathrm{~mm} \times 4.0 \mathrm{~mm} \times 3.2 \mathrm{~mm}$. Magnets and iron parts are alternately arranged. Magnetization and current directions are shown in Fig. 4. DC and AC for driving and position estimation are applied.

Figure 5 shows the structure of mover and stator. The mover consists of additional mover and mover for driving. The mover for driving part has 200 turns and the directions of current become alternate clockwise and counterclockwise rotation per each stage. The additional mover for position estimation has 15 turns. Position estimation of the additional mover is explained in Section 2-3. The total number of turns of the mover is 215 .

The stators consist of inner stator and outer stators. The stators are mainly made of magnets and iron which are alternately arranged. Permalloy is placed at the core of the additional mover for position estimation. The stators are placed in a multi-layer structure and laterally stacked alternately with mover. This structure increases the magnetic flux linking the 

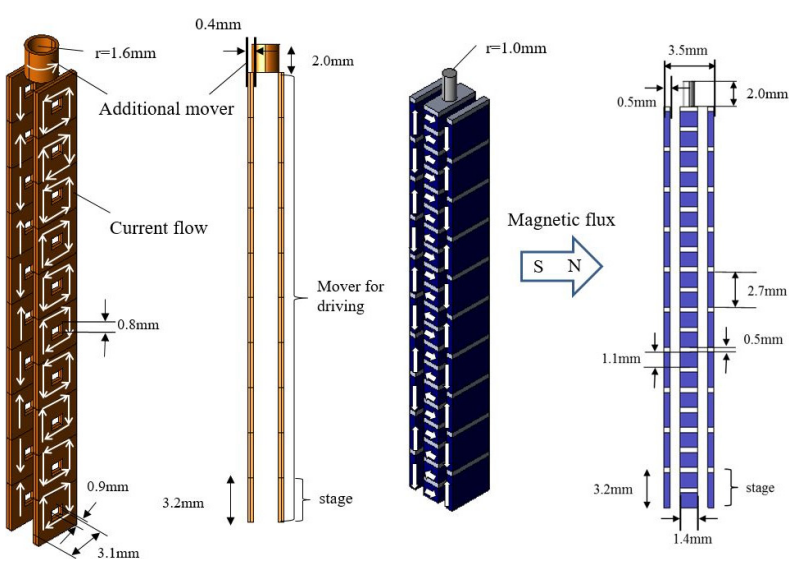

Fig. 5. Structure of mover and stator

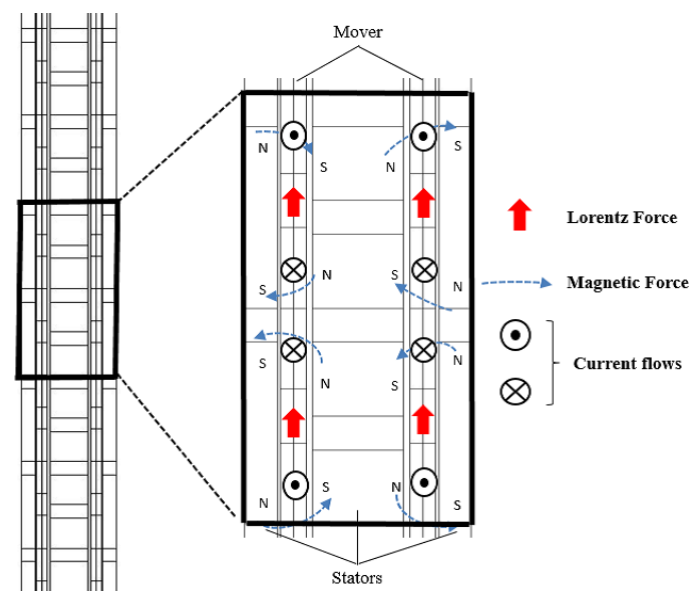

Fig. 6. Directions of magnetic flux, Lorentz force and current

mover. Thus, the output force increases compared with previous actuator ${ }^{(11)}$. The shape of the outer stator is a square tube to enable the actuators to be arranged in a plane easily.

2.2 Principle of Lorentz Force Generation This section explains the principle of force generation. Figure 6 shows the directions of the magnetic flux, Lorentz force, and current in the mover. The magnetic flux crosses through the movers as shown in Fig. 6. Lorentz force is generated by the product in the current in the movers and the magnetic flux. Therefore, this actuator can realize a pushing motion and a pulling motion by changing the directions of current.

The Lorentz force of the mover $F_{y}$ is calculated as

$$
\begin{gathered}
F=\int_{V}(\boldsymbol{J} \times \boldsymbol{B}) d V, \ldots \ldots \ldots \ldots \ldots \ldots \ldots \\
F_{y}=\int_{0}^{x_{1}} \int_{0}^{l} \int_{0}^{d} \frac{2 n I}{d\left(x_{1}-x_{2}\right)} B_{z}(x, y, z) d x d y d z,
\end{gathered}
$$

where $\boldsymbol{J}, \boldsymbol{B}, V, B_{z}(x, y, z)$, and $n$ denote the vector of current density, the vector of magnetic flux density, the area where magnetic flux and movers cross, the magnetic flux density of $\mathrm{z}$-axis direction and the number of turns of the coil. $x_{1}, x_{2}, l$, and $d$ define as shown in the Fig. 4 .

2.3 Principle of Mover Position Estimation This section proposes a method to use the inductance of the actuator to detect the mover position. The inductance of the actuator is calculated from AC current and AC voltage applied

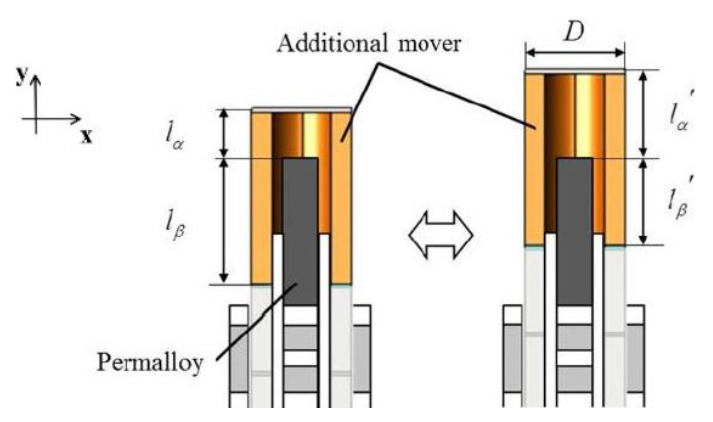

(a) Displacement: $0 \mathrm{~mm}$

(b) Displacement: $1 \mathrm{~mm}$

Fig. 7. Cross-section view of additional mover and stator (Permalloy)

to the movers. This section explains the relation between the inductance and displacement.

Inductance of the actuator $L$ is expressed as

$$
L=L_{N}+L_{A}
$$

where $L_{N}$ and $L_{A}$ denote inductance of the mover for driving, and inductance of the additional mover. The ratio of the inductance $L_{A}$ to the inductance of the actuator $L$ is large since magnetic flux of the additional mover is larger than that of the mover for driving.

Figure 7 shows a cross-section view of the mover for position estimation. Figure 7(a) is a cross-section view without displacement and Fig. 7(b) is a cross-section view with displacement.

$L_{A}$ is calculated as

$$
L_{A}=L_{\alpha}+L_{\beta},
$$

where $L_{\alpha}$ and $L_{\beta}$ denote inductance of an air core coil and inductance of a permalloy core coil. Permalloy has high permeability and can increase inductance. $L_{\alpha}$ and $L_{\beta}$ are expressed as

$$
\begin{aligned}
& L_{\alpha}=K_{\alpha} \mu_{0} N^{2}\left(\frac{\pi D^{2}}{4}\right) l_{\alpha}, \cdots \\
& L_{\beta}=K_{\beta} \mu_{0} \mu_{r} N^{2}\left(\frac{\pi D^{2}}{4}\right) l_{\beta},
\end{aligned}
$$

where $K_{\alpha}, K_{\beta}, \mu_{0}, \mu_{r}, N, D, l_{\alpha}$, and $l_{\beta}$ denote the Nagaoka coefficient of the air core coil, the Nagaoka coefficient of the permalloy core coil, permeability of vacuum, relative permeability of permalloy, the number of turns per length, the diameter of the mover, the length of the air core coil, and the length of the permalloy core coil.

The permeability of permalloy is very large compared with that of vacuum. Therefore, relation of inductance of an air core coil and inductance of a permalloy core coil is expressed as

$$
L_{\alpha}<L_{\beta}
$$

The length of the permalloy core coil $l_{\beta}$ decreases when displacement of the mover is increased. Therefore, $L_{A}$ decreases. Relation of inductance of the additional winding is expressed as

$$
L_{A}>L_{A}^{\prime}, \cdots
$$

where $L_{A}$ and $L_{A}^{\prime}$ denote the inductance of Fig. 7(a) and the 
Table 3. Simulation parameter in thermal analysis

\begin{tabular}{c|c}
\hline Parameter & Value \\
\hline Thermal conductivity of magnet & $7.0 \mathrm{~W} /(\mathrm{m} \cdot \mathrm{K})$ \\
Specific heat of magnet & $410 \mathrm{~J} /(\mathrm{kg} \cdot \mathrm{K})$ \\
Density of magnet & $7.50 \mathrm{~g} / \mathrm{cm}^{3}$ \\
Thermal conductivity of copper & $400 \mathrm{~W} /(\mathrm{m} \cdot \mathrm{K})$ \\
Specific heat of copper & $380 \mathrm{~J} /(\mathrm{kg} \cdot \mathrm{K})$ \\
Density of copper & $8.69 \mathrm{~g} / \mathrm{cm}^{3}$ \\
Thermal conductivity of iron & $50 \mathrm{~W} /(\mathrm{m} \cdot \mathrm{K})$ \\
Specific heat of iron & $480 \mathrm{~J} /(\mathrm{kg} \cdot \mathrm{K})$ \\
Density of iron & $7.80 \mathrm{~g} / \mathrm{cm}^{3}$ \\
Coefficient of heat transfer & $5 \mathrm{~W} /\left(\mathrm{m}^{2} \cdot \mathrm{K}\right)$ \\
Air temperature & $20^{\circ} \mathrm{C}$ \\
Internal resistance of movers & $1.24 \Omega$ \\
\hline \multicolumn{2}{c}{}
\end{tabular}

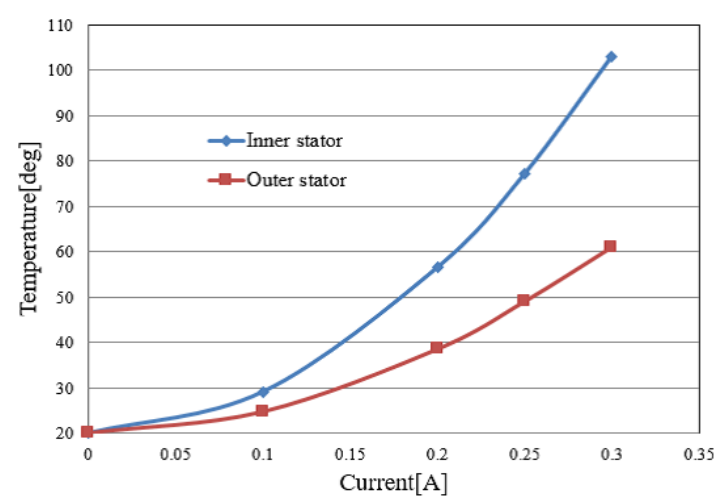

Fig. 8. Relation between current and temperature of actuator

inductance of Fig. 7(b). Accordingly, the inductance of the actuator $L$ in 3 decreases when displacement of the mover is increased because of the influence of $L_{A}$ in 4 .

Moreover, the inductance of the actuator $L$ is calculated as

$$
L=\frac{1}{2 \pi f} \sqrt{\left(\frac{V_{a m p}}{I_{a m p}}\right)^{2}-\left(R+R_{s}\right)^{2}},
$$

where $f$ denote the frequency ${ }^{(5)}$. Thus, if voltage and current amplitude of the actuator $V_{a m p}$ and $I_{a m p}$ can be masesured, the mover position can be estimated.

\section{Thermal Analysis}

The recommended temperature of magnets is $80^{\circ} \mathrm{C}$ or lower since the performance of magnets decline by higher temperature. A large current will heats up the actuator. Therefore, it is necessary to consider the maximum allowable current. Temperatures of magnets and iron parts are calculated by simulation software. These simulations were conducted in a steady state and a transient state by using a magnetic field analysis of the electromagnetic analysis software, "JMAG Designer" based on the finite element method. Simulation parameters in thermal analysis are listed in Table 3. They are set as thermal conductivity, specific heat, and density. Air temperature is assumed to be $20^{\circ} \mathrm{C}$. Electric power consumption $P$ is calculated as

$$
P=I^{2} R
$$

where $I$ and $R$ denote current of the movers and internal resistance of the movers.

Figure 8 shows the relation between current and temperature of the actuator. It indicates the thermal equilibrium values when constant DC current is applied to the actuator. The

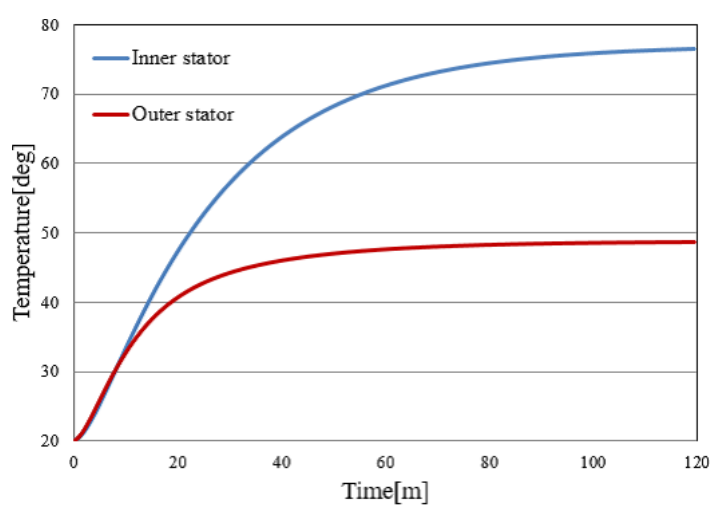

Fig. 9. Relation between time and temperature of the actuator $(0.25 \mathrm{~A})$

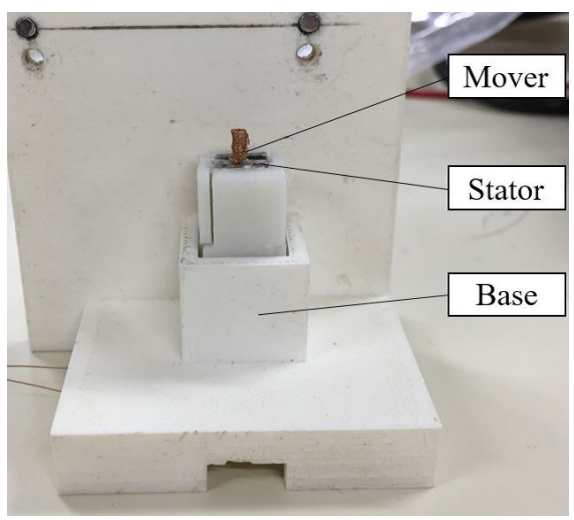

Fig. 10. Fabricated actuator

horizontal axis is current and the vertical axis is temperatures of the inner stator, and outer stator. The temperature of the inner stator is higher than temperature of the outer stator. The temperature of the inner stator was higher than $80^{\circ} \mathrm{C}$ when the current was over $0.25 \mathrm{~A}$. Additionally, Fig. 9 shows the relation between time and temperature of the actuator $(0.25 \mathrm{~A})$. it indicates that the time untill the temperature of inner stator reach about $80^{\circ} \mathrm{C}$ takes between 100 and 120 minutes. Hence, DC current $0.25 \mathrm{~A}$ can be applied to the actuator.

\section{Experiments}

This section describes the experimental results. The experiments of output force measurement and inductance measurement were conducted to verify the validity of proposed actuator.

4.1 Fabrication of Actuator Figure 10 shows the fabricated actuator. A copper which a wire diameter is $0.18 \mathrm{~mm}$ was used for the mover. Since the shape of mover can not maintained with copper wire alone, the mover is fixed with an epoxy resin type adhesive. The stator parts are fixed to the plastic parts made with 3D printer.

4.2 Experimental System This section explains the experimental system.

The experimental system is shown in Fig. 11. The experimental parameter is in Table 4. A high speed digital control system called 'PE-Expert4' made by Myway plus Corporation was used. The main $\mathrm{CPU}$ is a digital signal processor (DSP). The A/D converter samples the date every $8 \mu \mathrm{s}$. The $\mathrm{D} / \mathrm{A}$ converter outputs a signal every $8 \mu \mathrm{s}$. The AC $500 \mathrm{mV}$ voltage is used for position estimation which is generated by 


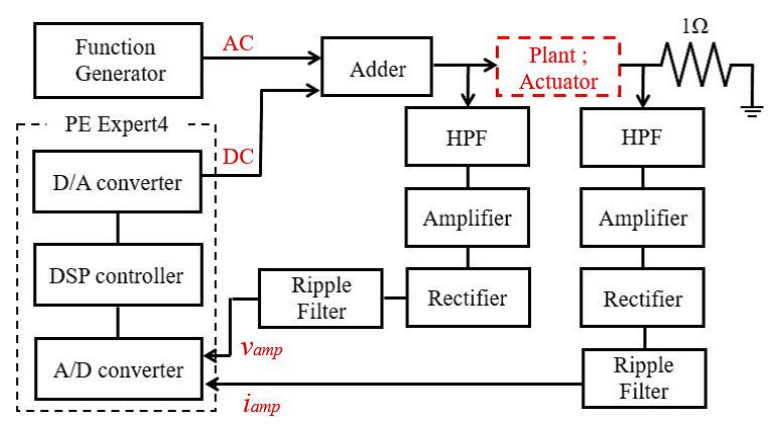

Fig. 11. Experimental system

Table 4. Experimental parameter

\begin{tabular}{c|c}
\hline Cut-off frequency of HPF & $1.59 \mathrm{kHz}$ \\
Initial position (Mover) & $0.3 \mathrm{~mm}$ \\
Shunt resistance & $1.0 \Omega$ \\
Gain of amplifier & 8.18 \\
Amplitude of the AC voltage & $500 \mathrm{mV}$ \\
Frequency of the AC signal & $100 \mathrm{kHz}$ \\
Amplitude of the DC voltage & $650 \mathrm{mV}$ \\
Sampling time of A/D converter & $8 \mu \mathrm{s}$ \\
Sampling time of D/A converter & $8 \mu \mathrm{s}$ \\
\hline
\end{tabular}

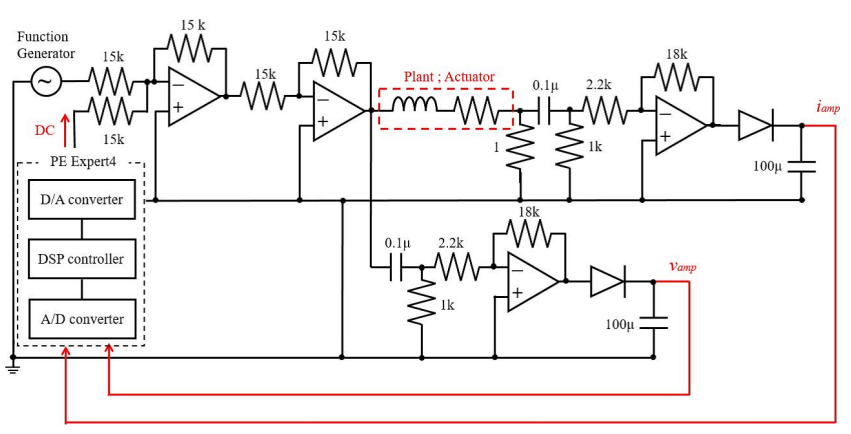

Fig. 12. Experimental circuit

a function generator with $100 \mathrm{kHz}$. And the DC voltage and DC currents are $650 \mathrm{mV}$ and $0.25 \mathrm{~A}$ respectively and they generate the thrust force.

The controller generates the digital signals. The digital signals pass through the D/A converter, and adder circuit. The signals are changed to a voltage including AC and DC components by adder circuit and applied to the actuator. DC components is not needed for estimation of the accurate positon. Hence, DC components are removed by high-pass filter after passing through the actuator. The cut-off frequency was set to $1.59 \mathrm{kHz}$. The gain of amplifier was set to 8.18 .

Moreover, it is difficult to measure the signal of $100 \mathrm{kHz}$ by the 'PE-Expert4', because the minimum sampling time is $5 \mu \mathrm{sec}$. Therefore, the signals to A/D converter are smoothed by a rectifier and a filter circuit. To measure the current, a shunt resistance $R_{s}=1 \Omega$ is connected to the actuator in series. The actuator has the inductance of the actuator $L$ and an internal resistance $R=1.24 \Omega$.

The experimental circuit is shown in Fig. 12.

4.3 Output Force Measurement This section explains the experiment of output force measurement. Figure 13 shows the experimental equipment. The XYZ stage and load cell which is fixed to XYZ stage severally measures displacement and output force of the actuator. The digital indicator is used to display output force. Specifications of the load cell, and the digital indicator are listed in Table 5.

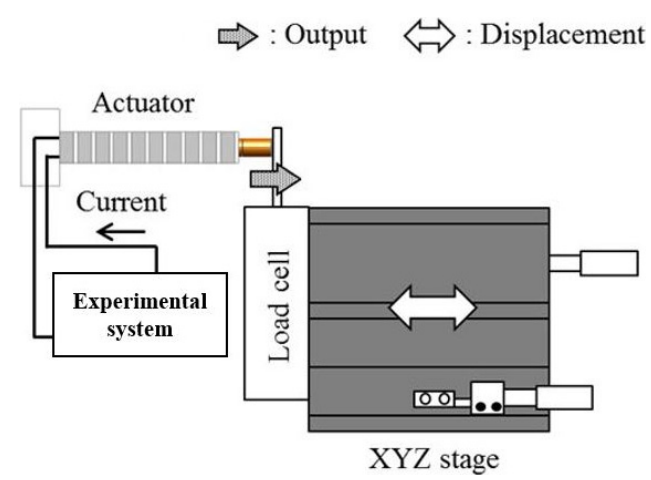

Fig. 13. Experimental equipment

Table 5. Specifications of equipment

\begin{tabular}{c|c|c}
\hline Equipment & Load cell & Digital indicator \\
\hline Model & ULA-10GR & CSD-815B \\
Maker & Minebea Co., Ltd. & Minebea Co., Ltd. \\
Repeatability & $0.1961 \mathrm{mN}$ & - \\
Rating & $98.07 \mathrm{mN}$ & - \\
\hline
\end{tabular}

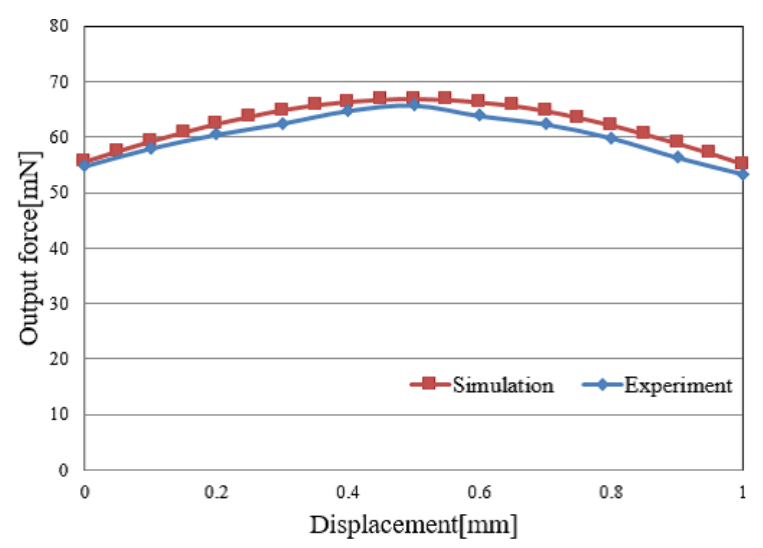

Fig. 14. Experimental results and simulation results of Lorentz force

Figure 14 shows the experimental results and the simulation results of the Lorentz force. The horizontal axis is displacement of the mover and the vertical axis is the Lorentz force of the actuator. Displacement of the mover is from $0.0 \mathrm{~mm}$ to $1.0 \mathrm{~mm}$. The maximum Lorentz force is $66.9 \mathrm{mN}$. The experimental result almost coincides with the JMAG simulation result ${ }^{(15)}$

Figure 15 shows relation between maximum output force per unit area and displacement of proposed actuator and various actuators. The proposed actuator has better performance in term of output force per unit area and displacement between $0 \mathrm{~mm}$ and $1.0 \mathrm{~mm}$ compared with other actuators. Therefore, the proposed actuator is superior as an actuator for tact-cell.

4.4 Inductance Measurement This section explains the experiment of inductance measurement. The 'PEExpert4' measures voltage and current amplitude of the actuator $V_{a m p}$ and $I_{a m p}$. The laser sensor measures displacement of the mover.

Figure 16 shows the relation between the $I_{a m p}, V_{a m p}$ and displacement. The horizontal axis is displacement of the mover and the vertical axis is the voltage and current amplitude of the actuator.

The inductance is estimated using by the data of Fig. 16 


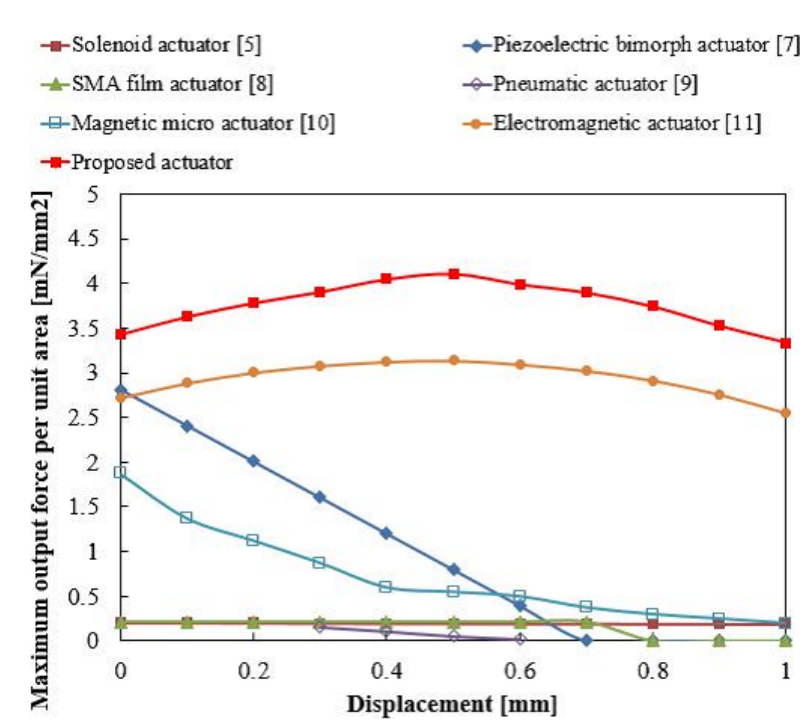

Fig. 15. Relation between maximum output force and displacement of proposed actuator and various actuators

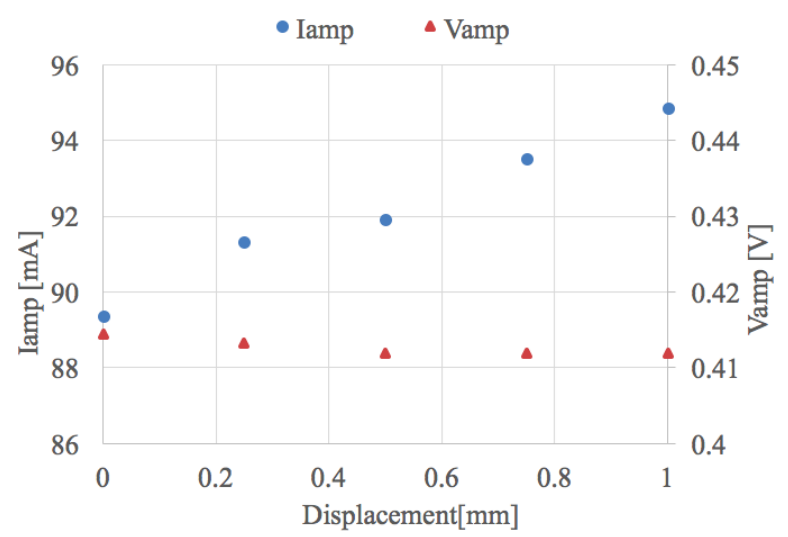

Fig. 16. Relation between $I_{a m p}, V_{a m p}$ and displacement. (Experiment)

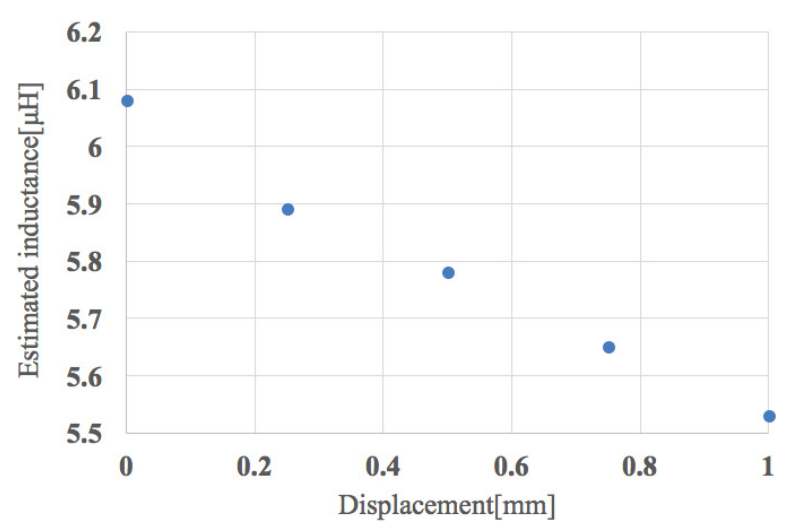

Fig. 17. Relation between estimated inductance and displacement. (Experiment)

and (9). Figure 17 shows the relation between the estimated inductance and displacement. Inductance of the actuator $L$ is decreased when displacement is increased. Therefore, the mover position can be estimated by the voltage amplitude and the current amplitude of the actuator.

\section{Conclusion}

This paper focused on a novel compact actuator aiming at realization of a two-dimensional tactile display. The proposed electromagnetic linear actuator can accurately control the position and force, while being compact and lightweight as a tact-cell. The $3 \mathrm{D}$ size of the actuator is $4.0 \mathrm{~mm} \times 4.0 \mathrm{~mm}$ $\times 34.0 \mathrm{~mm}$. The verification experiments were conducted with the system that DC and AC voltage are applied to actuator simultaneously. The result of output force measurement shows that the proposed actuator has better performance in term of output force per unit surface area and mover stroke between $0 \mathrm{~mm}$ and $1.0 \mathrm{~mm}$ compared with other actuators. Further the result of inductance measurement shows that the proposed actuator can be sensorlessly controlled by using the voltage and current amplitude of the actuator.

\section{References}

( 1 ) K. Ohnishi, S. Katsura, and T. Shimono: "A Compact Broadband Tactile Display and Its Effectiveness in the Display of Tactile Form", IEEJ Trans. Industrial Electronics Magazine., Vol.4, No.2, pp.16-19 (2010)

( 2 ) T. Shimono, Y. Hatta, and N. Motoii: "otal Harmonic Distortion and Content Rate of Spatial Modal Information for Haptic Parallel Motion Analysis", AUTOMATIKA., Vol.54, No.1, pp.39-48 (2013)

( 3 ) S. Katsura, W. Yamanouchi, and Y. Yokokura: "Real-World Haptics: Reproduction of Human Motion", IEEJ Trans. IE, Vol.6, No.1, pp.25-31 (2012)

( 4 ) K. Ohnishi, T. Shimono, and K. Natori: "Haptics for medical applications", Artificial Life and Robotics., Vol.13, No.2, pp.383-389 (2009)

( 5 ) S. Nagai, T. Nozaki, and A. Kawamura: "Real-time Sensorless Estimation of Position and Force for Solenoid Actuators", IEEJ Trans. IA, Vol.5, No.2, pp.32-38 (2016)

( 6 ) S. Nagai and A. Kawamura: "Realization of Bilateral Control by Compact Solenoid Actuators without Position and Force Sensors", 14th IEEE International Workshop on Motion Control, AMC2016, pp.1-6, Auckland, New Zealand (2016)

( 7 ) K.-U. Kyung, M. Ahn, D.-S. Kwon, and M.A. Srinivasan: "A Compact Broadband Tactile Display and Its Effectiveness in the Display of Tactile Form", in Proc. of IEEE Proc. 1st Joint Eurohaptics Conf. Haptic Interfaces Visual Environ. Teleoperator Syst., pp.600-601 (2005)

( 8 ) R. Vitushinsky, S. Schmitz, and A. Ludwig: "Bistable Thin-Film Shape Memory Actuators for Applications in Tactile Displays", IEEE J. Microelectromech. Syst., Vol.18, No.1, pp.186-194 (2009)

( 9 ) X. Wu, S.-H. Kim, H. Zhu, C.-H., and M.G. Allen: "A Refreshable Braille Cell Based on Pneumatic Microbubble Actuators", IEEE J. Microelectromech. Syst., Vol.21, No.4, pp.908-916 (2012)

(10) J. Streque, A. Talbi, P. Pernod, and V. Preobrazhensky: "New Magnetic Microactuator Design Based on PDMS Elastomer and MEMS Technologies for Tactile Display”, IEEE Trans. Haptics, Vol.3, No.2, pp.88-97 (2010)

(11) S. Masuyama, T. Noguchi, and A. Kawamura: "One stator made of two parts and one rotor electromagnetic linear actuator for tactile display", IEEJ Trans. Industry Applications, Vol.6, No.2, pp.160-164 (2017)

(12) A. Russomanno, S. O'Modhrain, R.B. Gillespie, and M.W.M. Rodger: “Refreshing Refreshable Braille Displays", IEEJ Trans. on Haptics, Vol.8, No.3, pp.287-297 (2015)

(13) Y. Kato, T. Sekitani, M. Takamiya, M. Doi, K. Asaka, T. Sakurai, and T. Someya: "Sheet-Type Braille Displays by Integrating Organic Field-Effect Transistors and Polymeric Actuators", IEEJ Trans. on Electron Devices, Vol.54, No.2, pp.202-209 (2007)

(14) S. Nagai, T. Nozaki, and A. Kawamura: "Environmental Robust Position Control for Compact Solenoid Actuators by Sensorless Simultaneous Estimation of Position and Force", IEEE Trans. Industrial Electronics, Vol.63, No.8, pp.5078-5086 (2016)

(15) T. Noguchi and A. Kawamura: "A Novel Small Linear Actuator for Tactile Display Using High Density Magnetic Flux", in Proc. of IEEE Int. Conf. on Electrical Machines and Systems. ICEMS2016, pp.1-5 (2016) 
Takuya Noguchi (Student Member) received the B.E. degrees in elec-

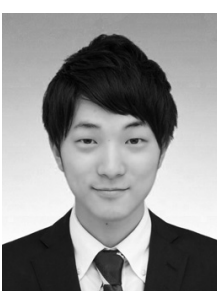
trical and computer engineering from Yokohama National University, Kanagawa Japan, in 2016. He is now a master course student of Yokohama National University. He has belonged to the Kawamura laboratory since April 2015. His reserch interests include linear actuator, sensorless control and haptics.

Sakahisa Nagai

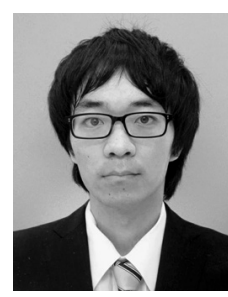

(Student Member) received the B.E. and M.E. degrees in electrical and computer engineering from Yokohama National University, Kanagawa Japan, in 2014 and 2016, respectively, where he is currently working toward the Ph.D. degree. He has been with the Kawamura laboratory at Yokohama National University since April 2013. His research interests includes sensorless actuation and motion control.
Atsuo Kawamura (Fellow) received the Ph.D. degree in electrical en-

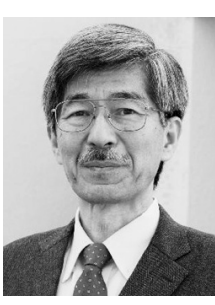
gineering from the University of Tokyo in 1981. After the five-year-stay at the University of MissouriColumbia as a faculty member, he joined the department of electrical and computer engineering at okohama National University in 1986, and now he is a professor. His interests are in the fields of power electronics, digital control, electric vehicles, train traction control and robotics. He received Transaction Paper Award from IEEE in 1988, 2001 and 2002, also from IEE of Japan in 1996. Dr. Kawamura is an IEEE Fellow, and Fellow of the IEE of Japan. He is also a researcher in Kanagawa Institute of Industrial Science and Technology. 